\title{
Where to Take a Rest: An Attention Restoration Theory Perspective
}

\author{
Kyung-mi Chung ${ }^{1,2}$, Jin Young Park ${ }^{1,2}$ \\ ${ }^{1}$ Department of Psychiatry, Gangnam Severance Hospital, Yonsei University Health System \\ Seoul, South Korea \\ ${ }^{2}$ Department of Psychiatry and Institute of Behavioral Science in Medicine, Yonsei University College of Medicine \\ Seoul, South Korea \\ chungkyungmi@yuhs.ac; empathy@yuhs.ac
}

\section{Extended Abstract}

The purpose of the present study is to test the following research questions: RQ1. How does individuals' presence experience during exposure to $360^{\circ}$ virtual nature differently affect the subjective evaluation of perceived restorativeness? RQ2. How does individuals' presence experience during exposure to $360^{\circ}$ virtual nature differently affect involuntary attention restoration as indicated by the modulation of auditory Mismatch Negativity (aMMN) and P3a amplitude?; RQ3. Will the 'subjective' self-reported and 'objective' event-related potential (ERP) responses be congruent? Based on attention restoration theory [1], nature can allow depleted directed attention to rest and restore by drawing involuntary attention that requires no efforts. According to previous ERP studies on the during-effect of meditation, long-term mediators showed significantly reduced aMMN [2] and P3a [3] amplitudes than non-mediators. Assuming that the differences in individual's presence level would boost this meditation-like effect [4] and the aMMN would be attention-independent [5], we hypothesized that people with a high level of presence experience would have significantly increased perceived restorativeness scale (PRS) scores and reduced P3a responses to the natural virtual environment (VE) than those with a low level of presence experience.

A total of forty healthy volunteers (22 males), aged 19 to 36 years $(M=23.78, S E=.56)$, were enrolled in this experiment. The $360^{\circ}$ nature video was chosen from Google YouTube and then edited (Size: $4 \mathrm{~K}, 3840 \times 1920$ pixels; Running time: 5 m $53 \mathrm{~s}$ ). While actively viewing the video with LG VR glasses compatible with a LG G5 smartphone on, participants were guided to concentrate only on the give visual task and to ignore task-irrelevant incoming sounds via headphones. A passive auditory oddball paradigm was designed to last for $5 \mathrm{~m} 30 \mathrm{~s}$ and composed of $750-\mathrm{Hz}$ standard (180 trials; $75 \mathrm{~dB})$ and 1000$\mathrm{Hz}$ deviant (60 trials; $75 \mathrm{~dB}$ ) tones and the inter-stimulus-interval randomly presented between $1000 \mathrm{~ms}$ and $1300 \mathrm{~ms}$. After the video ended, all participants were asked to fill out the PRS [6] and presence questionnaires [7, 8].

An independent samples $t$-test was performed to compare the PRS scores and MMN/P3a complex amplitudes between the two groups divided by a median split on the presence scale: (1) low-presence group [If $\mathrm{PQ}=<84, N=21 ; M=72.90, S E$ $=2.39$ ] and (2) high-presence group [If $84<\mathrm{PQ}, N=19 ; M=97.63, S E=2.02$ ]. It was found that when viewing the natural VE, the high presence group $(M=110.84, S E=5.59)$ showed significantly higher PRS scores than the low presence group $(M=92.57, S E=4.83), t(38)=-2.487, p<.05)$, but both aMMN $[t(38)=.808, N S]$ and P3a $[t(38)=.930, N S]$ amplitudes were not significantly lower in the high presence group.

In conclusion, individual's presence experience failed to strengthen the restorative effect of exposure to virtual nature, and their self-reported and ERP responses to virtual nature experience appeared to be incongruent. These findings might be explained by the possibility of unsatisfied full sensorial richness or satisfied presence sensation in the $360^{\circ} \mathrm{VE}$.

\section{Acknowledgement}

This article is part of the first author's doctoral dissertation submitted to Sungkyunkwan University. This work was supported by the National Research Foundation of Korea (NRF) grant funded by the Korea government (MSIP) (No. 2016R1C1B2010739).

\section{References}


[1] S. Kaplan, "The restorative benefits of nature: Toward an integrative framework," Journal of environmental psychology, vol. 15, pp. 169-182, 1995.

[2] B. Biedermann, P. De Lissa, Y. Mahajan, V. Polito, N. Badcock, M. H. Connors, et al., "Meditation and auditory attention: An ERP study of meditators and non-meditators," International Journal of Psychophysiology, vol. 109, pp. 63-70, 2016.

[3] B. R. Cahn and J. Polich, "Meditation (Vipassana) and the P3a event-related brain potential," International Journal of Psychophysiology, vol. 72, pp. 51-60, 2009.

[4] Y. De Kort, A. Meijnders, A. Sponselee, and W. IJsselsteijn, "What's wrong with virtual trees? Restoring from stress in a mediated environment," Journal of environmental psychology, vol. 26, pp. 309-320, 2006.

[5] R. Näätänen, A. W. Gaillard, and S. Mäntysalo, "Early selective-attention effect on evoked potential reinterpreted," Acta psychologica, vol. 42, pp. 313-329, 1978.

[6] T. Hartig, F. G. Kaiser, and P. A. Bowler, Further development of a measure of perceived environmental restorativeness: Institutet för bostads-och urbanforskning, 1997.

[7] B. G. Witmer and M. J. Singer, "Measuring presence in virtual environments: A presence questionnaire," Presence, vol. 7, pp. 225-240, 1998.

[8] B. G. Witmer, C. J. Jerome, and M. J. Singer, "The factor structure of the presence questionnaire," Presence: Teleoperators \& Virtual Environments, vol. 14, pp. 298-312, 2005. 